Fluxos de Anosov de codimensão um que são suspensões

Renato Alejandro Tintaya Mollo 

SERVIÇO DE PÓS-GRADUAÇÃO DO ICMC-USP

Data de Depósito: 17 de junho de 2009

Assinatura:

\title{
Fluxos de Anosov de codimensão um que são suspensões
}

\author{
Renato Alejandro Tintaya Mollo
}

Orientador: Prof. Dr. Carlos Alberto Maquera Apaza

Dissertação apresentada ao Instituto de Ciências Matemáticas e de Computação - ICMC-USP, como parte dos requisitos para obtenção do título de Mestre em Ciências - Matemática.

USP - São Carlos

Junho/2009 

A minha amada mãe Basilia, e os meus irmãos Manuel e Jesús, pela saudade de ficar longe, ao meu saudoso pai Jesús Manuel in memorian. 



\section{Agradecimentos}

Agradeço primeiramente a Deus, pelo dom da vida e por ter me dado forças para concluir mais esta etapa dos meus estudos.

Um agradecimento especial ao meu orientador professor Carlos Alberto Maquera Apaza pela sua eficiente orientação, paciência, boa vontade, sabedoria, pelo exemplo de dedicação à profissão e por ter aceitado me orientar.

A minha familia, pelo apoio incondicional e por terem sido a força que me fez ir em frente, por estar sempre presentes .

Ao Walter pelo apoio nos momentos difíceis e ajuda neste trabalho.

Aos meus professores do ICMC, Denise, Márcia, Daniel, Eugenio, Plácido por me incentivarem a continuar nos estudos.

Ao meus colegas e grandes amigos: Jaqueline, Iris e Pedro ("Ceará"). Por ter me ajudado a superar as dificuldades de estar longe de casa.

Aos amigos do mestrado do ICMC, que de alguma forma me ajudaram a nunca desistir: todos os meninos e meninas das salas 4-007, 4-008 e 4-009.

Aos funcionários do ICMC, pela atenção e convivência amiga durante a realização do curso.

Ao professor Walter Torres Montes da Universidad Nacional de San Agustín-Arequipa pela dedicação ao ensino da matemática e incentivo constante de ser o melhor a cada día.

Ao meus grandes amigos que fiz durante minha estadia em São Carlos, Roxana, Soledad, Edwin, Cesar, Waldo.

À CAPES ( Coordenação de Aperfeiçoamento de Pessoal de Ensino Superior) pelo apoio financiero.

E a todos meus amigos que sempre estiveram presente, de alguma maneira, me encorajaram, ajudaram ou torceram por mim. Dentre eles, Pathy, Alvaro, Claudio, Claudia, Enrique, Anita, Laura, Ingrid, Christian, Sandra e outros.

Obrigado a todos. 



\section{Resumo}

O objetivo principal desta dissertação é mostrar um resultado obtido por Plante em [12] o qual estabelece que: qualquer fluxo de Anosov de codimensão um sobre uma variedade diferenciável compacta $M$ de dimensão maior do que 3 com grupo fundamental solúvel é topologicamente equivalente à suspensão de um automorfismo hiperbólico do toro.

Este resultado mostra a conjectura de Verjovsky no caso que o grupo fundamental da variedade é um grupo solúvel. A prova deste resultado é baseada no celebre resultado de Schwartzman [15], Teorema 2.17, o qual fornece um criterio para garantir a existencia de seção transversal global para um fluxo não singular. 



\section{Abstract}

The aim of this dissertation is to show an result obtained by Plante, in [12], which establishes that: Any codimension one Anosov flow on a closed manifold $M$ of dimension greater than 3 and fundamental group solvable is topologically equivalent to a suspension of a hyperbolic toral automorphism.

This result shows the Verjovsky's conjecture in the case that the manifold has fundamental group solvable. The proof of this result is based in the celebrated result of Schwartzman [15], Teorema 2.17, which provides a criterion for existence of global cross section for an non singular flow. 



\section{Sumário}

Introdução

1 Preliminares 3

1.1 Folheações: noções e resultados fundamentais . . . . . . . . . . . . . . 3

1.2 Fluxos de Anosov . . . . . . . . . . . . . . . . . . . . . . 9

1.2.1 Partições de Markov de um Fluxo de Anosov . . . . . . . . . . . . . 12

2 O critério de Schwartzman

2.1 Seção transversal global e suspensões . . . . . . . . . . . . . . . . . . 15

2.2 Ciclos assintóticos . . . . . . . . . . . . . . . . . 16

2.3 O critério de Schwartzman . . . . . . . . . . . . . . . . 21

3 Grupo fundamental solúvel e Existência de seção transversal global: Um Teorema de Plante

3.1 Demonstração do Teorema Principal . . . . . . . . . . . . . . . . . . . 26 



\section{Introdução}

Os sistemas de Anosov (difeomorfismo ou fluxo de Anosov) tem um papel central na teoria dos sistemas dinâmicos, estes sistemas representam a idéia mais perfeita do comportamento hiperbólico global. Esta noção tem sido introduzida por D.V. Anosov nos anos 60's em [1]. Existe uma extensa literatura focada no estudo de classificação de certos sistemas de Anosov. Em particular, por um resultado celebre de Newhouse [11] e Franks [6], cada difeomorfismo de Anosov de codimensão um sobre uma variedade compacta é topologicamente mixing (mais do que transitivo). Como um corolário deste teorema e [6, Corollary (6.4)], a menos de recobrimentos finitos, difeomorfismos de Anosov de codimensão um sobre uma variedade compacta de dimensão $\geq 3$ são topologicamente conjugados a automorfismos hyperbolicos do toro. Para fluxos, no caso 3-dimensional, Franks-Williams [7] construíram um fluxo de Anosov que não é topologicamente transitivo. No casos de dimensão > 3, em 1974 Verjovsky [19] provou que fluxos de Anosov de codimensão um sobre uma variedade fechada de dimensão maior do que 3 são transitivos e Conjeturou: fluxos de Anosov de codimensão um numa variedade de dimensão maior do que 3 são topologicamente equivalentes à suspensão de um automorfismo hiperbólico do toro. No caso tridimensional, Bonatti-Langevin construíram um fluxo de Anosov que não é suspensão.

O objetivo principal desta dissertação é mostrar um resultado obtido por Plante em [12] o qual mostra a conjectura de Verjovsky no caso que o grupo fundamental da variedade é um grupo solúvel. Mais precisamente.

Teorema 3.1 Qualquer fluxo de Anosov de codimensão um sobre uma variedade diferenciável compacta $M$ de dimensão maior do que 3 e de classe $C^{\infty}$ com grupo fundamental solúvel é topologicamente conjugado à suspensão de um automorfismo hiperbólico do toro.

A prova deste resultado é baseada no celebre resultado de Schwartzman [15], Teorema 2.17, o qual fornece um criterio para garantir a existencia de seção transversal global para um fluxo não singular. Para isto ele estabelece uma correspondência $1-1$ entre as 
medidas de probabilidade invariantes pelo fluxo com o primeiro grupo de homologia da variedade. A classe de homologia associada a uma medida de probabilidade invariante pelo fluxo é chamada de ciclo assintótico. A condição do seu critério é em termos destas classes.

A estratégia para mostrar o Teorema 3.1, a grosso modo, sera a seguinte. Primeiramente, mostraremos que a condição do Teorema de Schwartzman é satisfeita, isto implicara que o fluxo admite uma seção transversal global $\Sigma$. Isto significa que a aplicação de primeiro retorno $T: \Sigma \rightarrow \Sigma$ é um difeomorfismo e o fluxo é topologicamente equivalente à suspensão de $T$. O fato do fluxo $\varphi^{t}$ ser de Anosov implica que a aplicação de primeiro retorno $T: \Sigma \rightarrow \Sigma$ é um difeomorfismo de Anosov, logo, pelo Teorema de Franks-Newhouse (Teorema 3.3), $T$ é topologicamente conjugado a um automorfismo hiperbólico do toro. Consequentemente, $\varphi^{t}$ é topologicamente equivalente à suspensão de um automorfismo hiperbólico do toro.

Esta dissertação esta organizada como segue. No primeiro capitulo é desenvolvida a teoria necessaria sobre folheações e fluxos de Anosov, a maioria das provas são omitidas. No segundo capitulo, primeiramente definiremos o que é uma seção transversal global e a sua relação com as suspensões (Seção 2.1), em seguida definiremos o que é um ciclo assintótico (Seção 2.2) para finalmente, na Seção 3.3, estabeleceremos o critério de Schwartzman, Teorema 2.17, o qual garante a existência de tal seção. Finalmente, usando partições de Markov associadas a um fluxo de Anosov, no terceiro capitulo mostramos o Teorema de principal desta dissertação. 


\section{Capítulo}

\section{Preliminares}

\subsection{Folheações: noções e resultados fundamentais}

Definição 1.1. Seja $M$ uma variedade de dimensão $m$ e classe $C^{\infty}$. Uma folheação de classe $C^{r}$ de dimensão $n$ de $M$, é um atlas máximo $\mathscr{F}$ de classe $C^{r}$ em $M$ tal que:

i) Se $(U, \phi) \in \mathscr{F}$ então $\phi(U)=U_{1} \times U_{2} \subset \mathbb{R}^{n} \times \mathbb{R}^{m-n}$, onde $U_{1}$ e $U_{2}$ são discos abertos de $\mathbb{R}^{n}$ e $\mathbb{R}^{m-n}$ respectivamente.

ii) Se $(U, \phi)$ e $(V, \psi) \in \mathscr{F}$ são tais que $U \cap V \neq \emptyset$, então a mudança de coordenadas $\psi \circ \phi^{-1}: \phi(U \cap V) \rightarrow \psi(U \cap V)$ é de classe $C^{r}$ e tem a forma, $\psi \circ \phi^{-1}(x, y)=$ $\left(h_{1}(x, y), h_{2}(y)\right)$, onde $(x, y) \in \mathbb{R}^{n} \times \mathbb{R}^{m-n}$.

Dizemos também que $M$ é folheada por $\mathscr{F}$, ou ainda que $\mathscr{F}$ é uma estrutura folheada de dimensão $n$ e de classe $C^{r}$ sobre $M$.

Folhas de uma folheação. Seja $\mathscr{F}$ uma folheação de classe $C^{r}$ e dimensão $n, 0$ $n<m$ de uma variedade $M^{m}$. Consideremos uma carta local $(U, \phi)$ de $\mathscr{F}$ tal que $\phi(U)=U_{1} \times U_{2} \subset \mathbb{R}^{n} \times \mathbb{R}^{m-n}$ os conjuntos da forma $\phi^{-1}\left(U_{1} \times\{c\}\right), c \in U_{2}$ são chamados de placas de $U$, ou ainda placas de $\mathscr{F}$.

Fixado $c \in U_{2}$ a aplicação $f=\left.\phi^{-1}\right|_{U_{1} \times\{c\}}: U_{1} \times\{c\} \rightarrow U$ é um mergulho de classe $C^{r}$, por tanto as placas são subvariedades conexas de dimensão $n$ e de classe $C^{r}$ de $M$. Além disto se $\alpha$ e $\beta$ são placas de $U$ então $\alpha \cap \beta=\emptyset$ ou $\alpha=\beta$.

Um caminho de placas de $\mathscr{F}$ é uma sequência $\alpha_{1}, \ldots, \alpha_{k}$ de placas de $\mathscr{F}$ tal que $\alpha_{j} \cap \alpha_{j+1} \neq \emptyset$ para todo $j \in\{1, \ldots, k-1\}$. Como $M$ é recoberta pelas placas de $\mathscr{F}$, 
definimos em $M$ a relação de equivalência:

$" p \sim q$ se existe um caminho de placas $\alpha_{1}, \ldots, \alpha_{k}$ com $p \in \alpha_{1}$ e $q \in \alpha_{k} "$.

As classes de equivalência da relação $\sim$ são chamadas folhas de $\mathscr{F}$. Segue da definição que uma folha de $\mathscr{F}$ é um subconjunto de $M$ conexo por caminhos.

Outro fato importante é que toda folha $F$ de $\mathscr{F}$ possui uma estrutura de variedade $C^{r}$ de dimensão $n$ naturalmente induzida pelas cartas de $\mathscr{F}$. A demonstração desta afirmação pode ser encontrada em [4], a continuação temos um exemplo de uma folheação de dimensão um.

\section{Exemplo 1.2. (campo de vetores sem singularidades)}

Seja $X$ um campo de vetores de classe $C^{r}, r \geq 1$, sobre uma variedade compacta $M$. Se $X$ não tem singularidades então ele define uma folheação de dimensão 1 sobre $M$ de classe $C^{r}$, cujas folhas são as órbitas do fluxo gerado pelo campo, isto é garantido pelo teorema do fluxo tubular.

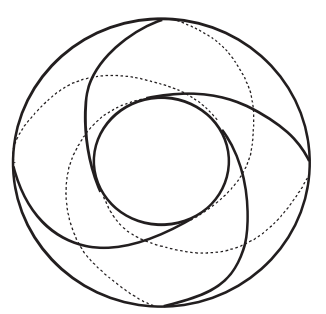

Figura 1.1: Folheação do Toro

Definição 1.3. Seja $\mathscr{F}$ uma folheação sobre a variedade $M$ então dizemos que o conjunto $S \subset M$ é saturado por $\mathscr{F}$ ou $\mathscr{F}$-saturado se $S$ é a união de folhas de $\mathscr{F}$.

Se demonstra em [4] que o fecho e o complementar de conjuntos saturados são saturados.

Definição 1.4. Dizemos que duas folheações $\mathscr{F}$ e $\mathscr{F}^{\prime}$ de uma variedade diferenciável $M$ são topologicamente equivalentes quando existe um homeomorfismo $H: M \rightarrow M$ que leva folhas de $\mathscr{F}$ em folhas de $\mathscr{F}^{\prime}$. O homeomorfismo $H$ é chamado de equivalência topológica entre $\mathscr{F}$ e $\mathscr{F}^{\prime}$.

Campos de Planos e Folheações. A partir de agora até o fim deste capitulo, a menos que seja especificado outra coisa, vamos supor $\mathscr{F}$ é uma folheação de classe $C^{r}, r \geq 1$ sobre a variedade compacta $M$ de classe $C^{\infty}$. 
Definição 1.5. (i) Um campo de $k$-planos sobre uma variedade $M^{m}, 1 \leq k \leq m$, é uma aplicação $P: M \rightarrow T_{x} M$ tal que $P(x)$ é um subespaço $k$-dimensional de $T_{x} M$ para cada $x \in M$.

(ii) O campo de $k$-planos $P$ é de classe $C^{r}$ se para todo $x \in M$ existe uma vizinhança $U$ sobre a qual são definidos $k$ campos de vetores de classe $C^{r}$ linearmente independentes $X_{1}, \ldots, X_{k}: U \rightarrow T U$ tais que $P(x)$ é gerado por $\left\{X_{1}(x), \ldots, X_{k}(x)\right\}$ para cada $x \in U$.

Quando $k=1$, $\mathrm{P}$ é chamado de campo de linhas.

Exemplo 1.6. Toda folheação $\mathscr{F}$ de classe $C^{r}$, com $r \geq 1$, de dimensão $k$ sobre uma $m$-variedade $M$ de classe $C^{\infty}$ define um campo de $k$-planos $T \mathscr{F}$ sobre $M$ de classe $C^{r-1}$ dado por

$$
T \mathscr{F}(x)=T_{x} F_{x}
$$

No exemplo anterior vemos que se temos uma folheação de dimensão $k$ é possível encontrar um campo de $k$-planos tangente à folheação. No entanto, a recíproca não é verdade.

Definição 1.7. Dizemos que um campo de $k$-planos $P$ de classe $C^{r}$ é integrável se $P=$ $T \mathscr{F}$ para alguma folheação $\mathscr{F}$ de classe $C^{r+1}$.

No que segue estabelecemos um resultado, devido a Frobenius, que garante a integrabilidade de um campo de $k$-planos. Antes disso precisamos fazer algumas considerações.

Se $X$ é um campo de vetores de classe $C^{r}, r \geq 1$ sobre uma variedade compacta $M$, então seu fluxo sera denotado por $X^{t}$.

Definição 1.8. Sejam $X$ e $Y$ campos de vetores de classe $C^{r}, r \geq 2$. O colchete de Lie de $X, Y$ é o campo de vectores $[X, Y]$ definido por

$$
[X, Y](p)=\left.\frac{d}{d t}\right|_{t=0}\left(X_{*}^{t}(Y)(p)\right), \quad \text { onde } X_{*}^{t}(Y)(p)=D X^{-t}\left(X^{t}(p)\right) \cdot Y\left(X^{t}(p)\right) \text {. }
$$

Se fixando coordenadas os campos $X$ e $Y$ são da forma

$$
X=\sum_{i} a_{i} \frac{\partial}{\partial x_{i}}, Y=\sum_{i} b_{i} \frac{\partial}{\partial x_{i}},
$$

então pode ser verificado que, nestas coordenadas, $[X, Y]$ tem a seguinte forma:

$$
[X, Y]=\sum_{i, j}\left(a_{i} \frac{\partial b_{j}}{\partial x_{i}}-b_{i} \frac{\partial a_{j}}{\partial x_{i}}\right) \frac{\partial}{\partial x_{j}} .
$$


Um campo de vetores $X$ é tangente ao campo de $k$-planos $P$, neste caso denotaremos por $X \in P$, se $X(x) \in P(x)$ para todo $x \in M$.

Definição 1.9. Dizemos que um campo de planos $P$ é involutivo quando $[X, Y] \in P$ toda vez que $X, Y \in P$.

A demonstração do seguinte lema pode ser encontrada em [14] ou [18].

Lema 1.10. Se $\mathscr{F}$ é uma folheação de classe $C^{r}, r \geq 1$, então o campo de planos T $\mathscr{F}$ é involutivo.

Uma questão que surge imediatamente é a seguinte. Qualquer campo de planos $P$ é da forma $P=T \mathscr{F}$ para alguma folheação $\mathscr{F}$ ?

Teorema 1.11 (Teorema de Frobenius). Seja $P$ um campo $k$-planos de classe $C^{r}, r \geq 1$. Se P é involutivo então é integrável.

A noção de folheação orientável e transversalmente orientável. Primeiramente, lembremos a noção de orientabilidade para um espaço vetorial. Dado um espaço vetorial $E$ de dimensão $(n \geq 1)$, dizemos que duas bases ordenadas de $E, \mathcal{B}=\left\{u_{1}, \ldots, u_{n}\right\}$ e $\mathcal{B}^{\prime}=\left\{v_{1}, \ldots, v_{n}\right\}$, definem a mesma orientação em $E$ se a matriz de mudança de base $A=\left(a_{i j}\right)_{1 \leq i, j \leq n}$, definida por $v_{i}=\sum_{j=1}^{n} a_{i j} u_{j}$, tem determinate positivo. Se $B$ é o conjunto das bases ordenadas de $E$, a relação " $\mathcal{B}$ e $\mathcal{B}^{\prime}$ definem a mesma orientação em $E$ " é uma relação de equivalência em $B$, a qual possui duas classes de equivalência chamadas as orientações de $E$.

Consideremos uma cobertura de $M$ por abertos $\left(U_{i}\right)_{i \in I}$ tal que, para cada $i \in I$, a restrição $P \mid U_{i}$ é definida por $k$ campos de vetores contínuos $X^{1}, \ldots, X^{k}$. Para cada $x \in U_{i}$, as bases $\mathcal{B}(x)=\left\{X^{1}(x), \ldots, X^{k}(x)\right\}$ e $\mathcal{B}^{\prime}(x)=\left\{-X^{1}(x), \ldots, X^{k}(x)\right\}$ definem duas orientações distintas de $P(x)$, as quais denotamos por $\mathcal{O}_{i}^{+}(x)$ e $\mathcal{O}_{i}^{-}(x)$, respectivamente.

Definição 1.12. Seja $P$ um campo de $k$-planos contínuo em $M$. Diremos que $P$ é orientável se para cada $x \in M$ for possível escolher uma orientação $\mathcal{O}(x)$ em $P(x)$ de tal forma que se $\mathcal{O} \mid U_{i}=\mathcal{O}_{i}^{+}$para todo $i$, então $\mathcal{O}_{i}^{+}=\mathcal{O}_{j}^{+}$sempre que $U_{i} \cap U_{j} \neq \emptyset$. Se $k=\operatorname{dim} M$ e $P(x)=T_{x} M$ dizemos que $M$ é orientável.

Por exemplo, um campo de linhas $P$ em $M$ é orientável se e somente se existe um campo de vetores contínuo $X$ em $M$ tal que, para todo $x \in M, P(x)$ é o subespaço gerado por $X(x)$. 
Sejam $\widehat{M}=\left\{\left(x, \mathcal{O}_{x}\right) \mid x \in M\right.$ e $\mathcal{O}_{x}$ é uma das orientações de $\left.P(x)\right\}$ e $\pi: \widehat{M} \rightarrow M$ a projeção $\pi\left(\left(x, \mathcal{O}_{x}\right)\right)=x$. Para cada $x \in M, \pi^{-1}(x)=\{(x, \mathcal{O}),(x,-\mathcal{O})\}$, onde $\mathcal{O}$ é uma das orientações em $P(x)$ e $-\mathcal{O}$ é a outra. Em $\widehat{M}$ consideramos a topologia cuja base de abertos é construída a seguir. Dado $\left(x_{0}, \mathcal{O}_{0}\right) \in \widehat{M}$, seja $U$ vizinhança de $x_{0}$ em $M$ onde está definida uma orientação contínua $\mathcal{O}$ de $P \mid U$. Podemos supor $\mathcal{O}_{x_{0}}=\mathcal{O}_{0}$. Definimos então uma vizinhança $V$ de $\left(x_{0}, \mathcal{O}_{0}\right)$ como $V=\left\{\left(x, \mathcal{O}_{x}\right) \mid x \in U\right\}$. Com esta topologia, $\pi: \widehat{M} \rightarrow M$ é um recobrimento de duas folhas (isto é, $\pi^{-1}(x)$ é um conjunto de dois elementos para todo $x \in M)$. Como $\pi$ é um homeomorfismo local podemos definir uma estrutura diferenciável $C^{\infty}$ em $\widehat{M}$, compatível com a topologia co-induzida por $\pi$ e tal que $\pi$ é um difeomorfismo local de classe $C^{\infty}$.

O recobrimento duplo orientável de $P$ é por definição o campo de $k$-planos $\pi^{*}(P)_{x}=$ $D \pi^{-1} P(\pi(x))$. Temos a seguinte definição.

Definição 1.13. Dizemos que uma folheação de classe $C^{r}(r \geq 1)$ e dimensão $n$ é orientável se o campo de $n$-planos $T \mathscr{F}$ é orientável. Similarmente, $\mathscr{F}$ é transversalmente orientável se existe um campo de $(m-n)$-planos complementar a $T \mathscr{F}$ que é orientável.

Observação 1.14. Dado $p \in M$ sempre existe uma seção transversal local de $\mathscr{F}$ passando por $p$.

De fato consideremos uma carta local $(U, \phi) \in \mathscr{F}$ com $p \in U, \phi(U)=U_{1} \times U_{2} \subset$ $\mathbb{R}^{n} \times \mathbb{R}^{s}, \phi(p)=\left(c_{1}, c_{2}\right)$ e tomarmos $\Sigma=\phi^{-1}\left(\left\{c_{1}\right\} \times U_{2}\right)$. Como $\left\{c_{1}\right\} \times U_{2}$ é transversal às placas $U_{1} \times c, c \in U_{2}$, segue que $\Sigma$ é uma seção transveral de $\mathscr{F}$.

Uma ferramenta importante no estudo qualitativo das folheações é o chamado grupo de holonomia, que definiremos a seguir.

Holonomia. Sejam $\mathscr{F}$ uma folheação sobre uma variedade $M$ e $U_{1}, \ldots, U_{k}$ uma família finita de cartas folheadas de $\mathscr{F}$ tais que cada placa de cada $U_{i}$ intercepta pelo menos uma placa de $U_{j}$. Denotemos por $\pi_{i}: U_{i} \rightarrow \Sigma_{i}$ a projeção ao longo das placas, onde cada $\Sigma_{i}$ é dado pela observação anterior. Então, para cada $x \in \Sigma_{i}$ tal que sua placa $\alpha_{x}$ intercepta $U_{j}$, podemos definir

$$
\begin{gathered}
f_{i, j}(x)=\pi_{j}\left(\alpha_{x}\right) . \\
f_{i, j}: \operatorname{Dom}\left(f_{i, j}\right) \subset \Sigma_{i} \rightarrow \Sigma_{j}
\end{gathered}
$$

aplicação de holonomia induzida pelas cartas folheadas $\left(U_{i}, \varphi_{i}\right),\left(U_{j}, \varphi_{j}\right)$, notemos que $\operatorname{Dom}\left(f_{i, j}\right)$ é um conjunto aberto em $\Sigma_{i}$. Supondo que $U_{i} \cap U_{i+1} \neq \emptyset$ para cada $i \in$ 
$\{1, \ldots, k-1\}$, podemos definir a aplicação de holonomia $f_{1, \ldots, k}: \operatorname{Dom}\left(f_{1, \ldots, k}\right) \subset \Sigma_{1} \rightarrow \Sigma_{k}$ por

$$
f_{1, \ldots, k}=f_{k-1, k} \circ f_{k-2, k-1} \circ \cdots \circ f_{1,2} .
$$

Agora, seja $F$ uma folha de $\mathscr{F}$ e $x, y \in F$. Por definição $F$ é conexa e assim existe uma curva $c:[0,1] \rightarrow F$ ligando $x$ e $y$. Esta curva pode ser coberta por um número finito de cartas folheadas $U_{1}, \ldots, U_{r}$ tais que $x \in U_{1}$ e $y \in U_{r}$ e toda placa de $U_{i}$ intersecta pelo menos uma placa de $U_{j}$ (para todo $i, j$ ). Sem perda de generalidade podemos assumir que $x \in \Sigma_{1}, y \in \Sigma_{r}$. A aplicação:

$$
f_{c}=f_{1, \ldots, r}
$$

é a holonomia induzida pela curva $c$. Observemos que $f_{c}(x)=y$ por definição. Podemos encontrar em [5] que

1. $f_{c}$ independe do cobrimento $U_{1}, \ldots, U_{r}$.

2. Se $c, c^{\prime} \subset F$ são homotópicos em $F$ com pontos inicial e final fixos, então $f_{c}=f_{c^{\prime}}$ num conjunto aberto de $\Sigma_{1}$ contendo $x$;

3. Quando $x=y$ obtemos uma representação

$$
\Phi: \pi_{1}(F, x) \rightarrow \operatorname{Germ}(\Sigma, x)
$$

dado por

$$
\Phi(\gamma)=\left[f_{c}\right]
$$

onde $c$ é um representante de $\gamma \in \pi_{1}(F, x), \Sigma$ é uma seção transversal de $\mathscr{F}$ que contém $x \in F \mathrm{e}$

$$
\operatorname{Germ}(\Sigma, x)=\{f: \operatorname{Dom}(f) \subset \Sigma \rightarrow \Sigma: f(x)=x\} / \sim
$$

é o germe de aplicações de classe $C^{r}$ induzidas pela relação de equivalência $f \sim g$ se e somente se $f$ e $g$ coincidem numa vizinhança de $x$.

Definição 1.15. Chamamos grupo de holonomia de $F$ ao grupo $\operatorname{Hol}(F, x)=\Phi\left(\pi_{1}(F, x)\right)$

O grupo $\operatorname{Hol}(F, x)$, a menos de conjugação diferencial, não depende de $x \in F$. Assim podemos definir $\operatorname{Hol}(F)=\operatorname{Hol}(F, x)$ para algum $x$. A folha $F$ tem ou não tem holonomia se $\operatorname{Hol}(F) \neq 0$ ou $\operatorname{Hol}(F)=0$. Uma folheação sem holonomia é uma folheação cujas folhas não tem holonomia. 


\subsection{Fluxos de Anosov}

Nesta seção abordaremos algumas noções e resultados da teoria de fluxos sobre variedades. Particularmente, a nossa ênfase será nos fluxos de Anosov. Muitos resultados serão somente enunciados, sendo omitidas as suas demonstrações, podendo estas ser encontradas em Verjovsky [20], Smale [17], [16], Katok [10] e Robinson [13].

Definição 1.16. Seja $M^{m}$ uma variedade diferenciável de classe $C^{\infty}$. Um fluxo de classe $C^{r}, r \geq 0$ sobre $M$ é uma aplicação $\varphi: \mathbb{R} \times M \rightarrow M$ de classe $C^{r}$ tal que:

$$
\begin{aligned}
& \text { 1. } \varphi^{0}=i d_{M} \\
& \text { 2. } \varphi^{t+s}=\varphi^{t} \circ \varphi^{s} \forall t, s \in \mathbb{R} \text {; }
\end{aligned}
$$

onde $\varphi^{t}(x)=\varphi(t, x)$.

O fluxo $\varphi^{t}$ de classe $C^{r}$ determina um campo $X$ sobre $M$ de classe $C^{r-1}$ definido por $X(x)=\left.\frac{d}{d t} \varphi^{t}(x)\right|_{t=0}$, quando $r \geq 1$, cujo fluxo coincide com $\varphi^{t}$.

Os fluxos de Anosov são um caso especial de fluxos, e tem sido muito estudados. A continuação definiremos o que são os fluxos de Anosov e veremos algumas propriedades importantes.

Definição 1.17. Um fluxo de Anosov de classe $C^{r}$ numa variedade Riemanniana $M$ é um fluxo $\varphi^{t}: M \rightarrow M$ não singular, de classe $C^{r}, r \geq 1$ com as seguintes propriedades:

i) O fibrado tangente $T M$ se escreve como a soma de Whitney de três subfibrados vectoriais contínuos:

$$
T M=E^{s s} \oplus E^{c} \oplus E^{u u},
$$

tais que tanto $E^{s s}$ e $E^{u u}$ são invariantes pela diferencial $D \varphi^{t}$ para todo $t \in \mathbb{R}$ e $E^{c}$ é o fibrado de linhas tangentes a $X$.

ii) Existem constantes $A>0, B>0$ e $\lambda>0$ tais que

$$
\begin{gathered}
\left\|\left(D \varphi^{t}\right)_{x}(v)\right\| \leq A e^{-\lambda t}\|v\| \\
\left\|\left(D \varphi^{-t}\right)_{x}(w)\right\| \leq B e^{-\lambda t}\|w\|
\end{gathered}
$$

para todo $x \in M, v \in E_{x}^{s s}, w \in E_{x}^{u u}$ e $t \in \mathbb{R}$.

Chamamos os fibrados $E^{s s}$ e $E^{u u}$ de fibrados fortemente estável e fortemente instável, respectivamente. Agora denotaremos a soma direta $E^{s s} \oplus E^{u u}$ por $E^{s u}$. Definimos também 
o centro estável $E^{s}=E^{c} \oplus E^{s s}$ e o centro instável $E^{u}=E^{c} \oplus E^{u u}$. Seja $X$ a notação do campo vectorial cujo fluxo é $\varphi^{t}$, isto é $X(x)=\left.\frac{d}{d t} \varphi^{t}(x)\right|_{t=0}$.

Observação 1.18. Pugh-Shub em [9] mostraram que os fibrados $E^{s s}, E^{u u}, E^{s}$ e $E^{u}$ são integráveis. Denotemos por $\mathcal{W}^{s s}, \mathcal{W}^{u u}, \mathcal{W}^{s}$ e $\mathcal{W}^{u}$ as folheações definidas por estes fibrados, respectivamente, além disso denotamos por $W_{x}^{u u}, W_{x}^{s s}, W_{x}^{u}$ e $W_{x}^{s}$ a folha de $\mathcal{W}^{u u}, \mathcal{W}^{s s}$, $\mathcal{W}^{u}$ e $\mathcal{W}^{s}$ passando por $x$

Definição 1.19. Seja $N$ uma variedade diferenciável de dimensão $n$ e classe $C^{\infty}$, e seja $f: N \rightarrow N$ um difeomorfismo, consideremos o espaço quociente:

$$
M=N \times[0,1] / \sim
$$

onde $\sim$ é a relação de equivalência $(x, 1) \sim(f(x), 0)$.

O campo vetorial $\partial / \partial t$ em $N \times[0,1]$ define um campo em $M$ cujo fluxo é chamado de suspensão de $f$. Este fluxo é de classe $C^{r}$.

Definição 1.20. Dizemos que um difeomorfismo $f: N \rightarrow N$ de classe $C^{r}, r \geq 1$, é um difeomorfismo de Anosov sobre uma variedade $N$ se existe uma decomposição contínua do fibrado tangente $T N=E^{u} \oplus E^{s}$ tal que:

1. Ambos $E^{u}$ e $E^{s}$ são invariantes pela derivada $D f$;

2. Existem constantes $C>0$ e $0<\lambda<1$ tal que

$$
\begin{array}{ccc}
\left\|\left(D f^{n}\right)_{x}(v)\right\| \leq C \lambda^{n}\|v\| \quad v \in E_{x}^{s} & n>0 \\
\left\|\left(D f^{-n}\right)_{x}(w)\right\| \leq C \lambda^{n}\|w\| & w \in E_{x}^{u} & n>0 .
\end{array}
$$

Em [20] podemos encontrar o seguinte resultado.

Teorema 1.21. A suspensão de um difeomorfismo de Anosov é um fluxo de Anosov.

Exemplo 1.22. a) Automorfismo hiperbólico do toro: Seja $A$ uma matriz hiperbólica (i. e. o módulo de cada autovalor é diferente de um) $n \times n$ com entradas inteiras e determinante igual a 1 . Então $A$, como um isomorfismo linear de $\mathbb{R}^{n}$, preserva o reticulado $\mathbb{Z}^{n}$. Logo $A$ induz um difeomorfismo $h$ do toro $\mathbb{T}^{n}=\mathbb{R}^{n} / \mathbb{Z}^{n}$ que é chamado de Automorfismo hiperbólico do toro.

Segue, da Definição 1.20, que $h$ é um difeomorfismo de Anosov. Conseqüentemente pelo Teorema 1.21, a suspensão $\varphi^{t}$ de $h$ é um fluxo de Anosov sobre $\mathbb{T}^{n}$. 
b) Seja $S$ uma superfície Riemanniana compacta com curvatura seccional estritamente negativa, e seja $M$ o fibrado tangente unitário de $S$. Então o fluxo geodésico $g_{t}$ de $S$ restrito a $M$ é un fluxo de Anosov.

Um teorema clássico é o teorema da variedade estável para difeomorfismos de Anosov, que nos dá informações topologicas sobre as folheações definidas pelas distribuções de planos do fibrado tangente.

Notemos que o subfibrado $E^{u u}$ ( resp. $E^{s s}$ ) depende da parametrização do tempo do fluxo. Logo a folheação fortemente instável $\mathcal{W}^{u u}$ (resp. a fortemente estável $\mathcal{W}^{s s}$ ) também depende da parametrização do fluxo. Mas o subfibrado $E^{u}\left(\operatorname{resp} . E^{s}\right.$ ) não depende da parametrização do fluxo, assim a folheação centro estável e centro instável independem da parametrização do fluxo.

Uma folha $V$ de $\mathcal{W}^{u}$ é folheada por $\mathcal{W}^{u u}$ e pelo fluxo.

Definição 1.23. Dizemos que um fluxo de Anosov é de codimensão um se um dos fibrados $E^{s s}$ ou $E^{u u}$, tem dimensão um.

Trocando $\varphi^{t}$ por $\varphi^{-t}$, podemos supor, sem perda de generalidade, que $\operatorname{dim} E^{u u}=1$, ou seja, $\operatorname{dim} E^{c} \oplus E^{s s}=n-1$.

Definição 1.24. Seja $x \in M$ dizemos que $x$ é um ponto errante para o fluxo $\varphi^{t}$ se existe uma vizinhança $U$ de $x$ e un número $T>0$ tal que se $t \geq T$ então $\varphi^{t}(U) \cap U=\emptyset$. Em outro caso dizemos que $x$ é um ponto não errante.

Note que:

$$
\varphi^{t}(U) \cap U=\emptyset \Rightarrow U \cap \varphi^{-t}(U)=\emptyset .
$$

Denotaremos ao conjunto de pontos não errantes do fluxo $\varphi^{t}$ por $\Omega\left(\varphi^{t}\right)$ ou simplesmente por $\Omega$.

Agora consideremos a questão da densidade das variedades $W_{x}^{s s}, W_{x}^{u u}, W_{x}^{s}$ e $W_{x}^{u}$ em $M$. Podemos encontrar o seguinte Teorema em [19].

Teorema 1.25. Se $\varphi^{t}: M \rightarrow M$ é um fluxo de Anosov de codimensão um sobre uma variedade compacta de dimensão maior do que tres, então $\Omega\left(\varphi^{t}\right)=M$.

A demonstração do seguinte Teorema pode ser encontrada em [1].

Teorema 1.26. Se $\varphi^{t}$ é um fluxo de Anosov de codimensão um sobre uma variedade de dimensão maior do que três, então o conjunto dos pontos periódicos de $\varphi^{t}$ é denso em $\Omega$.

Em particular, o conjunto das órbitas periódicas é denso em $M$.

Mostraremos o seguinte resultado, como corolário do Teorema 1.25: 
Corolário 1.27. Se o conjunto das órbitas periódicas é denso em $M$ para um fluxo de Anosov de codimensão um sobre uma variedade conexa de dimensão maior do que três, então $W_{x}^{u}$ e $W_{x}^{s}$ são densas em $M$ para cada $x \in M$.

Demonstração. É suficiente mostrar que $\overline{W_{x}^{u}}=M$ para cada $x \in M$. Seja $x \in M$ e considere o conjunto saturado por $\mathcal{W}^{u}$ de $\overline{W_{x}^{u}}$. Já que estamos supondo $M$ conexa é suficiente mostrar que $\overline{W_{x}^{u}}$ é aberto em $M$. Seja $N$ uma vizinhança produto de $z \in \overline{W_{x}^{u}}$ e $p \in N$ um ponto periódico. Como $\overline{W_{x}^{u}}$ é invariante por $\varphi^{t}$ e $W_{p}^{s} \cap W_{z}^{u} \neq \emptyset$ temos que $p \in \overline{W_{x}^{u}}$. Desde que os pontos periódicos são densos em $N$ e $\overline{W_{x}^{u}}$ é fechado, concluimos que $N \subset \overline{W_{x}^{u}}$, isto é, $\overline{W_{x}^{u}}$ é aberto.

\subsubsection{Partições de Markov de um Fluxo de Anosov}

Estudaremos as partições de Markov associadas a um fluxo de Anosov $\varphi^{t}$ sobre uma variedade fechada $M$. Fixemos um ponto $x \in M^{n}$ escolhamos um disco aberto de codimensão um $D^{n-1}$ com centro em $x$ e transversal ao fluxo $\varphi^{t}$. Seja $D^{u}$ (respectivamente $\left.D^{s}\right)$ a folha passando por $x$ da folheação $\left.\mathcal{W}^{u}\right|_{D^{n-1}}\left(\left.\mathcal{W}^{s}\right|_{D^{n-1}}\right.$ respectivamente). Escolhamos um subconjunto $Q^{u}$ em $D^{u}\left(Q^{s}\right.$ em $\left.D^{s}\right)$ tal que o interior $\operatorname{Int}\left(Q^{u}\right)$ seja conexo (respectivamente $\operatorname{Int}\left(Q^{s}\right)$ seja conexo) contendo $x$ e que $\overline{\operatorname{Int}\left(Q^{u}\right)}=Q^{u}$ (respectivamente $\left.\overline{\operatorname{Int}\left(Q^{s}\right)}=Q^{s}\right)$. Suponhamos que o diâmetro de $Q^{u}$ (respectivamente $Q^{s}$ ) é menor que um número $\epsilon>0$ dado.

Definição 1.28. Seja $R=Q^{u} \times Q^{s}$ o subconjunto de $D^{n-1}$ cujos elementos são pontos de intersecção de uma folha de $\left.\mathcal{W}^{u}\right|_{D^{n-1}}$, passando por um ponto de $Q^{s}$, com uma folha de $\left.\mathcal{W}^{s}\right|_{D^{n-1}}$ passando por um ponto de $Q^{u}$. O conjunto $R$ é chamado de retângulo.

Denotamos por $\operatorname{Int}(R)$ o interior de $R$ em $D^{n-1}$. Para um ponto $x \in R$, denotamos por $R^{u}(x)$ (respectivamente $R^{s}(x)$ ) a folha de $W^{u} \mid R$ (respectivamente $W^{s} \mid R$ ) passando por $x$. Também denotamos por $\operatorname{Int}\left(R^{u}(x)\right)=R^{u}(x) \cap \operatorname{Int}(R)$ (respectivamente $\operatorname{Int}\left(R^{s}(x)\right)=$ $\left.R^{s}(x) \cap \operatorname{Int}(R)\right)$

Seja $\mathcal{R}=\left\{R_{i}\right\}_{i=1}^{r}$ uma familia disjunta de retângulos. Denotemos por:

$$
|\mathcal{R}|=\cup_{i=1}^{r} R_{i}
$$

Suponhamos, além disto, que toda órbita positiva intercepta $|\mathcal{R}|$. Para todo ponto $x \in$ $|\mathcal{R}|$, seja $\tau(x)$ o menor número real positivo tal que $\varphi^{\tau(x)}(x) \in|\mathcal{R}|$. A aplicação

$$
\tau:|\mathcal{R}| \rightarrow \mathbb{R}
$$


é chamada tempo de primeiro retorno, e a aplicação

$$
f:|\mathcal{R}| \rightarrow|\mathcal{R}|
$$

definida por $f(x)=\varphi^{\tau(x)}(x)$ é chamada aplicação de primeiro retorno. Em geral $\tau$ e $f$ não são continuas, mas $f$ é uma bijeção.

Definição 1.29. A familia disjunta $\mathcal{R}$ é chamada partição de Markov de tamanho $\varepsilon$ para o fluxo $\varphi^{t}$ se as seguintes condições são satisfeitas:

i) Existe uma número positivo $\tau_{1}$ com a seguinte propriedade: para todo $x \in M$, existe $t \in\left[0, \tau_{1}\right]$ tal que $\varphi^{t}(x) \in|\mathcal{R}|$.

ii) Para qualquer $i$ e $j$, o conjunto $\operatorname{Int}\left(R_{i}\right) \cap f^{-1}\left(\operatorname{Int}\left(R_{j}\right)\right)$ é conexo caso este ser não vazio, e $\tau$ é continua sobre $R_{i} \cap f^{-1}\left(R_{j}\right)$.

iii) Se $x \in \operatorname{Int}\left(R_{i}\right) \cap f^{-1}\left(\operatorname{Int}\left(R_{j}\right)\right)$, então $f$ aplica $\operatorname{Int}\left(R_{i}^{s}(x)\right)$ no $\operatorname{Int}\left(R_{j}^{s}(f(x))\right)$ e $f^{-1}$ aplica $\operatorname{Int}\left(R_{j}^{u}(f(x))\right)$ no $\operatorname{Int}\left(R_{i}^{u}(x)\right)$.

A seguir estabelecemos algumas propriedades de partições de Markov associadas a fluxos de Anosov. O seguinte teorema é devido a R. Bowen [3].

Teorema 1.30. Todo fluxo de Anosov admite uma partição de Markov de tamanho arbitrariamente pequeno.

Para cada partição de Markov $\mathcal{R}=\left\{R_{i}\right\}_{i=1}^{r}$ definimos uma matriz $r \times r A=\left(a_{i j}\right)$ com $a_{i j}=1$ se $\operatorname{Int} R_{i} \cap f^{-1}\left(\operatorname{Int} R_{j}\right) \neq \emptyset$ e 0 em outro caso. $A$ é chamada a matriz de transição.

Uma sequencia, $\mathbf{i}=\left\{i_{n}\right\}_{n=-\infty}^{n=\infty}$ com $i_{n} \in\{1,2, \ldots, r\}$ é chamada admissivel se para todo $n, a_{i_{n}, i_{n+1}}=1$. Esta sequencia admissível $\mathbf{i}_{n=-\infty}^{n=\infty}=\left\{i_{n}\right\}$ é cíclica se existe um inteiro positivo $q$ tal que $i_{n+q}=i_{n}$ para todo $n$, neste caso denotamos por

$$
\mathbf{i}=\left\{i_{0}, i_{1}, \cdots, i_{q-1}\right\}
$$

Uma sequencia cíclica $\left\{i_{0}, i_{1}, \cdots, i_{q-1}\right\}$ é chamada minimal se todos os indices $i_{j}$ são distintos. Tem-se as seguintes propriedades:

- A uma órbita de $\varphi^{t}$ corresponde uma sequência admissível.

- A uma órbita periódica corresponde uma sequência admissível cíclica.

- O número de sequências cíclicas minimais é finito. 
Seja

$$
\tau_{0}=\inf \{\tau(x)|x \in| \mathcal{R} \mid\}
$$

Temos o seguinte teorema.

Teorema 1.31. 1. Para qualquer sequência admissivel $\mathbf{i}=\left\{i_{n}\right\}$ existe um único ponto $x \in|\mathcal{R}|$ com a seguinte propriedade: existe uma sequência de tempos $\left\{t_{n}\right\}_{n=-\infty}^{\infty}$ tal que $t_{0}=0$ e $\tau_{0} \leq t_{n+1}-t_{n} \leq \tau_{1}$ para todo $n$ e $\varphi^{t_{n}}(x) \in R_{i_{n}}$.

2. Reciprocamente dado um ponto $x \in|\mathcal{R}|$, existe um número finito de sequências admissiveis com as propriedades acima. 


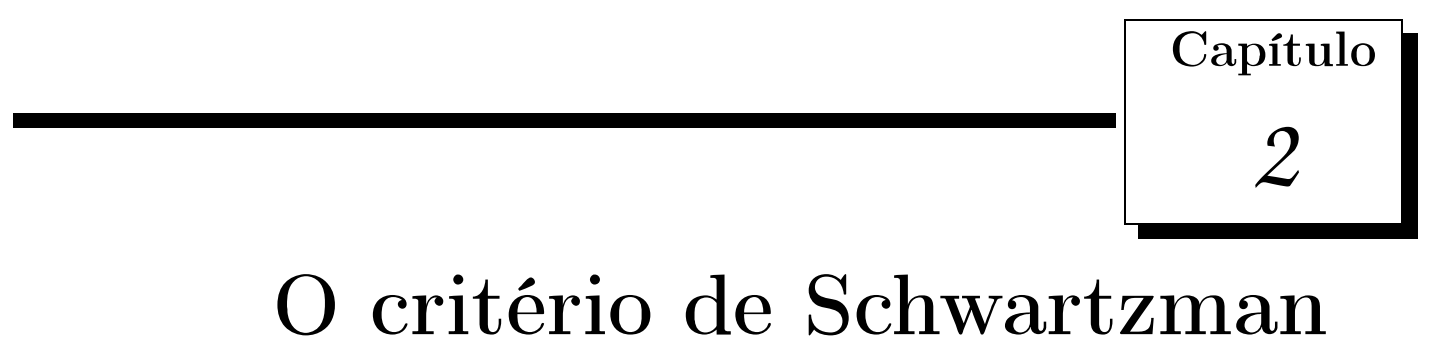

O teorema do fluxo tubular garante que um fluxo não singular sempre tem seções transversais locais; no entanto, uma seção transversal global é uma característica muito restritiva sobre o fluxo, mas nos dá muita informação da dinâmica do fluxo. Neste capítulo consideraremos um fluxo não singular $\varphi^{t}, t \in \mathbb{R}$, de classe $C^{r}, r \geq 1$, sobre uma variedade fechada $M$ de classe $C^{\infty}$. Em 1957 S. Schwartzman [15] publica um revolucionário artigo onde proporciona um critério que garante a existência de uma seção transversal global para um fluxo não singular. Para isto ele estabelece uma correspondência $1-1$ entre as medidas de probabilidade invariantes pelo fluxo com o primeiro grupo de homologia da variedade. A classe de homologia associada a uma medida de probabilidade invariante pelo fluxo é chamada de ciclo assintótico. A condição do seu critério é em termos destas classes.

Primeiramente definiremos o que é uma seção transversal global e a sua relação com as suspensões (Seção 2.1), em seguida definiremos o que é um ciclo assintótico (Seção 2.2) para finalmente, na Seção 2.3, estabeleceremos o critério de Schwartzman, Teorema 2.17, o qual garante a existência de tal seção.

\subsection{Seção transversal global e suspensões}

Definição 2.1. Chamamos de seção transversal global ao fluxo $\varphi^{t}$ a uma $C^{1}$ sub-variedade mergulhada $\Sigma \subset M$ de codimensão um tal que:

i) O fluxo é transversal a $\Sigma$.

ii) Cada órbita do fluxo intercepta $\Sigma$. 
Observação 2.2. Seja $\Sigma$ uma seção transversal global ao fluxo $\varphi^{t}$.

1. Existe uma aplicação $\tau: \Sigma \rightarrow \mathbb{R}^{+}$de classe $C^{1}$ tal que $\varphi^{\tau(x)}(x) \in \Sigma$ e para cada $t \in(0, \tau(x)), \varphi^{t}(x) \notin \Sigma$.

2. A aplicação $T: \Sigma \rightarrow \Sigma$ definida por $T(x)=\varphi^{\tau(x)}(x)$ é um difeomorfismo de classe $C^{1}$. Esta aplicação é chamada de Aplicação de primeiro retorno ou aplicação de Poincaré.

Os seguintes teoremas (os quais podem ser encontrados em [17] e [16]) mostram que se um fluxo admite uma seção transversal global, então alguns problemas para estes fluxos admitem uma redução para um problema de difeomorfismos numa dimensão a menos. Além disso cada difeomorfismo está associado a um difeomorfismo de uma seção transversal global de algum fluxo.

Teorema 2.3. Suponhamos que o fluxo $\varphi^{t}$ sobre $M$ admite uma seção transversal global $\Sigma$ e seja $T: \Sigma \rightarrow \Sigma$ a aplicação de primeiro retorno. Seja $\psi^{t}$ a suspensão do difeomorfismo T. Então $\psi^{t}$ é topologicamente equivalente a $\varphi^{t}$.

Entendemos que dois fluxos $\varphi^{t}: M \rightarrow M$ e $\psi^{t}: N \rightarrow N$ são topologicamente equivalentes quando existe um homeomorfismo $h: M \rightarrow N$ tal que $\psi^{t} \circ h=h \circ \varphi^{t}$.

Teorema 2.4. Seja $T: \Sigma \rightarrow \Sigma$ um difeomorfismo. Então o fluxo $\psi^{t}$, obtido pela suspensão de $T$ tem $\Sigma$ como seção transversal global com aplicação de primeiro retorno diferenciávelmente equivalente a $T$.

Entendemos que duas aplicações $T: \Sigma \rightarrow \Sigma$ e $H: \Gamma \rightarrow \Gamma$ são diferenciávelmente equivalentes quando existe um difeomorfismo $h: \Sigma \rightarrow \Gamma$ tal que $H \circ h=h \circ T$.

Teorema 2.5. Sejam $T_{0}: \Sigma_{0} \rightarrow \Sigma_{0}, T_{1}: \Sigma_{1} \rightarrow \Sigma_{1}$ difeomorfismos e $\varphi_{0}^{t}$ e $\varphi_{1}^{t}$ suas suspensões. Se $T_{0}$ e $T_{1}$ são topologicamente equivalentes então $\varphi_{0}^{t}$ e $\varphi_{1}^{t}$ são topologicamente equivalentes.

\subsection{Ciclos assintóticos}

Denotemos por $C(M)$ o conjunto das funções contínuas a valores reais e por $\mathcal{P}=\mathcal{P}(M)$ o espaço das medidas de probabilidade sobre $M$.

Sabemos que cada elemento $\mu$ de $\mathcal{P}$ pode ser considerado como um funcional linear $\mu: C(M) \rightarrow \mathbb{R}$ tal que:

1. $\mu(f) \geq 0$ para $f \geq 0 \mathrm{e}$, 
2. $\mu(1)=1$ onde $1 \in C(M)$ denota a função constante igual a 1 .

Definição 2.6. Uma medida de probabilidade $\mu$ sobre $M$ é dita invariante pelo fluxo ou simplesmente uma medida $\varphi$-invariante se

$$
\mu(A)=\mu\left(\varphi^{t}(A)\right), \quad \forall t \in \mathbb{R} \quad \text { e } \forall A \subset M \text { boreliano }
$$

Se os únicos conjuntos invariantes têm medida total ou medida nula com respeito a $\mu$, dizemos que $\mu$ é uma medida ergódica.

Denotemos por $\mathcal{P}_{\varphi}=\mathcal{P}_{\varphi}(M)$ o espaço das medidas de probabilidade $\varphi$-invariantes. Para definir ciclo assintótico, definiremos uma aplicação

$$
A: \mathcal{P}_{\varphi} \rightarrow H_{1}(M, \mathbb{R})
$$

a imagem de $\mu$ por $A$, a qual denotaremos por $A_{\mu}$, será chamado ciclo assintótico de $\mu$. Para tal propósito o seguinte resultado sera fundamental:

Proposição 2.7. O grupo de homologia $H_{1}(M, \mathbb{R})$ é isomorfo ao grupo $\operatorname{Hom}\left(\left[M, S^{1}\right], \mathbb{R}\right)$.

Onde $\left[M, S^{1}\right]$ denota o conjunto das classes de homotopia das aplicações contínuas de $M$ em $S^{1}$. Considerando $S^{1}$ como o grupo quociente $\mathbb{R} / \mathbb{Z}$, a estrutura de grupo aditivo de $S^{1}$ induz uma estrutura de grupo aditivo em $\left[M, S^{1}\right]$. Denotaremos por $C_{S^{1}}$ a classe fundamental de cohomologia de $S^{1}$. A proposição acima é consequência do seguinte resultado o qual pode ser encontrada em [8] e [21].

Proposição 2.8. $\quad$ i) A aplicação $\alpha:\left[M, S^{1}\right] \rightarrow H^{1}(M, \mathbb{R})$ é definida por $\alpha([g])=$ $g^{*}\left(C_{S^{1}}\right)$ e é um isomorfismo sobre $H^{1}(M ; \mathbb{Z})$.

ii) $H_{1}(M, \mathbb{R})$ é isomorfo a $\operatorname{Hom}\left(\left[M, S^{1}\right], \mathbb{R}\right)$.

Observação 2.9. Em virtude da Proposição 2.7, definir $A: \mathcal{P}_{\varphi} \rightarrow H_{1}(M, \mathbb{R})$ é equivalente a definir uma aplicação

$$
\bar{A}: \mathcal{P}_{\varphi} \times\left[M, S^{1}\right] \rightarrow \mathbb{R}
$$

tal que para cada $\mu$ em $P_{\varphi}$ a aplicação $A_{\mu}:=\bar{A}(\mu, \cdot):\left[M, S^{1}\right] \rightarrow \mathbb{R}$ é um homomorfismo.

A seguir definiremos a aplicação $\bar{A}$. Sejam $[g] \in\left[M, S^{1}\right]$ e $x \in M$ um elemento arbitrário de $M$. Seja $h_{x}: \mathbb{R} \rightarrow \mathbb{R}$ qualquer dos levantamentos da aplicação de $\mathbb{R}$ em $S^{1}$ cuja imagem de $t \in \mathbb{R}$ é dada por $g\left(\varphi^{t}(x)\right)$. Para $t \in \mathbb{R}$, definimos

$$
\Delta g(x, t)=h_{x}(t)-h_{x}(0)
$$


$\Delta g(x, t)$ mede a variação do ângulo do argumento de $g: M \rightarrow S^{1}$ ao longo do arco da órbita com extremos $x$ e $\varphi^{t}(x)$. A prova do seguinte lema é imediata.

Lema 2.10. Para g definida como acima temos:

i) $\Delta g(x, t)$ independe da escolha do levantamento e é continua em $x$ et.

ii) Temos $\Delta g(x, s+t)=\Delta g(x, s)+\Delta g\left(\varphi^{s}(x), t\right)$.

O item $i$ ) é consequência imediata das propriedades de levantamentos no círculo e o item $i i)$ é consequência da igualdade $\varphi^{t+s}=\varphi^{t} \circ \varphi^{s}$. Agora sim estamos em condições de definir $\bar{A}$.

Definição 2.11. Para cada $(\mu,[g]) \in \mathcal{P} \times\left[M, S^{1}\right]$ definimos

$$
\bar{A}(\mu,[g])=\frac{1}{t} \int_{M} \Delta g(x, t) d \mu(x), t \neq 0
$$

A aplicação $A_{\mu}=\bar{A}(\mu, \cdot)$ é chamado o ciclo assintótico de $\mu$.

Podemos dizer que $\bar{A}_{\mu}([g])$ mede o crescimento $\mu$-promedio do argumento de $g$ ao longo do fluxo numa unidade de tempo. A seguinte proposição mostra que $\bar{A}$ esta bem definida e que de fato $A_{\mu}$ representa uma classe em $H_{1}(M, \mathbb{R})$.

Proposição 2.12. A aplicação $\bar{A}$ esta bem definida e $A_{\mu}$ é um homomorfismo que independe da escolha de $g$ na sua classe e $t \neq 0$.

Demonstração. Primeiramente, mostraremos que a definição de $A_{\mu}([g])$ independe de $t$, para isto denotemos por

$$
B^{t}=\int_{M} \Delta g(x, t) d \mu(x)
$$

Usando o Lema 2.10 e a invariança da medida obtemos que

$$
\begin{aligned}
B^{s+t} & =\int_{M} \Delta g(x, s+t) d \mu(x) \\
& =\int_{M} \Delta g(x, s) d \mu(x)+\int_{M} \Delta g\left(\varphi^{s}(x), t\right) d \mu(x) \\
& =\int_{M} \Delta g(x, s) d \mu(x)+\int_{M} \Delta g(x, t) d \mu(x) \\
& =B^{s}+B^{t}
\end{aligned}
$$

Como consequência disto e da continuidade de $B^{t}$ temos que $B^{t}=t B^{1}$. Isto mostra que $A_{\mu}([g])$ independe de $t$. 
Sejam agora $[g],\left[g^{*}\right] \in\left[M, S^{1}\right]$ tais que $[g]=\left[g^{*}\right]$. Como a aplicação $g^{*}-g: M \rightarrow S^{1}$ é homotópicamente trivial, temos que esta aplicação tem um levantamento $h: M \rightarrow \mathbb{R}$. Logo:

$$
\Delta g^{*}(x, t)-\Delta g(x, t)=h\left(\varphi^{t}(x)\right)-h(x)
$$

Consequentemente, como $\mu$ é $\varphi^{t}$-invariante, temos que $A_{\mu}([g])=A_{\mu}\left(\left[g^{*}\right]\right)$.

Segue da definição que $A_{\mu}:\left[M, S^{1}\right] \rightarrow \mathbb{R}$ é um homomorfismo.

Observação 2.13. A aplicação $A: \mathcal{P}_{\varphi} \rightarrow H_{1}(M, \mathbb{R})$ é contínua e linear. Logo a sua imagem é um conjunto compacto e convexo.

Proposição 2.14. Se $\mu_{n} \in \mathcal{P}$ converge para uma medida de probabilidade invariante pelo fluxo $\mu \in \mathcal{P}_{\varphi}$, então

$$
A_{\mu}([g])=\lim _{n \rightarrow \infty} \frac{1}{t} \int_{M} \Delta g(x, t) d \mu_{n}(x)
$$

Demonstração. Se $\mu_{n} \in \mathcal{P}$ é tal que $\mu_{n} \rightarrow \mu$ na topologia fraca-*, então temos que

$$
\int_{M} f d \mu_{n}(x) \rightarrow \int_{M} f d \mu(x) \text { para toda } f \in C(M) \text { quando } n \rightarrow \infty .
$$

Em particular para $\Delta g(x, t)$, usando o Lema 2.10, temos que

$$
\begin{array}{r}
\int_{M} \Delta g(x, t) d \mu_{n}(x) \rightarrow \int_{M} \Delta g(x, t) d \mu(x) \\
\frac{1}{t} \int_{M} \Delta g(x, t) d \mu_{n}(x) \rightarrow \frac{1}{t} \int_{M} \Delta g(x, t) d \mu(x) .
\end{array}
$$

Portanto,

$$
A_{\mu}([g])=\lim _{n \rightarrow \infty} \frac{1}{t} \int_{M} \Delta g(x, t) d \mu_{n}(x)
$$

Definição 2.15. Para $p \in M$ e $T \in \mathbb{R}$, definimos uma medida de probabilidade $\mu_{p, T} \in \mathcal{P}$ dada por

$$
\mu_{p, T}(h)=\frac{1}{T} \int_{0}^{T} h\left(\varphi^{t}(p)\right) d t
$$

para qualquer $h \in C(M)$.

Observemos que $\mu_{p, T}$ não é necessariamente invariante pelo fluxo $\varphi^{t}$.

O seguinte resultado nos proporciona uma maneira de calcular $A_{\mu}([g])$. 
Lema 2.16. Suponhamos que para alguma sequência $p_{n}$ e $T_{n} \rightarrow \infty, \mu_{p_{n}, T_{n}}$ converge para $\mu$. Então $\mu$ é uma medida de probabilidade invariante, e temos

$$
A_{\mu}([g])=\lim _{n \rightarrow \infty} \frac{1}{T_{n}} \Delta g\left(p_{n}, T_{n}\right) .
$$

Demonstração. Pela proposição 2.14 temos:

$$
\begin{aligned}
A_{\mu}([g]) & =\lim _{n \rightarrow \infty} \frac{1}{t} \int_{M} \Delta g(x, t) d \mu_{p_{n}, T_{n}}(x) \\
& =\lim _{n \rightarrow \infty} \frac{1}{t} \mu_{p_{n}, T_{n}}(\Delta g(\cdot, t)) \\
& =\lim _{n \rightarrow \infty} \frac{1}{t} \frac{1}{T_{n}} \int_{0}^{T_{n}} \Delta g\left(\varphi^{s}\left(p_{n}\right), t\right) d s .
\end{aligned}
$$

Como $\Delta g\left(p_{n}, s+t\right)=\Delta g\left(\varphi^{s}\left(p_{n}\right), t\right)+\Delta g\left(p_{n}, s\right)$ temos

$$
\begin{aligned}
A_{\mu}([g]) & =\lim _{n \rightarrow \infty} \frac{1}{t} \frac{1}{T_{n}} \int_{0}^{T_{n}}\left(\Delta g\left(p_{n}, s+t\right)-\Delta g\left(p_{n}, s\right)\right) d s \\
& =\lim _{n \rightarrow \infty} \frac{1}{t} \frac{1}{T_{n}}\left(\int_{T_{n}}^{T_{n}+t} \Delta g\left(p_{n}, s\right) d s-\int_{0}^{t} \Delta g\left(p_{n}, s\right) d s\right)
\end{aligned}
$$

Agora observemos que a função $\Delta g(x, t)$ converge uniformemente em $x$ para $\Delta g(x, 0)=0$ quando $t \rightarrow 0$. Isto é, para qualquer $\epsilon>0$, existe $\delta>0$ tal que $|\Delta g(x, t)|<\epsilon$ sempre que $|t|<\delta$. Assim, junto com a igualdade

$$
\Delta g(x, s)-\Delta g(x, t)=\Delta g\left(\varphi^{t}(x), s-t\right)
$$

temos que; para $\epsilon>0$, existe $\delta>0$ (independente de $x, s$ e $t$ ) tal que $|\Delta g(x, s)-\Delta g(x, t)|$ $\epsilon$ sempre que $|s-t|<\delta$. Por tanto se $|t|<\delta$, então o termo

$$
\frac{1}{t}\left(\int_{T_{n}}^{T_{n}+t} \Delta g\left(p_{n}, s\right) d s-\int_{0}^{t} \Delta g\left(p_{n}, s\right) d s\right)
$$

pode ser aproximado por $\Delta g\left(p_{n}, T_{n}\right)$. Isto implica que

$$
\begin{aligned}
A_{\mu}([g]) & =\lim _{n \rightarrow \infty} \frac{1}{T_{n}} \frac{1}{t} \int_{0}^{T_{n}}\left(\Delta g\left(p_{n}, s+t\right)-\Delta g\left(p_{n}, s\right)\right) d s \\
& =\lim _{n \rightarrow \infty} \frac{1}{T_{n}} \Delta g\left(p_{n}, T_{n}\right) .
\end{aligned}
$$




\subsection{O critério de Schwartzman}

Agora daremos uma condição em termos de ciclos assintóticos para um fluxo admitir uma seção transversal global. O objetivo nesta seção é mostrar o seguinte resultado devido a Schwartzman.

Teorema 2.17. Seja $\varphi^{t}$ um fluxo não singular sobre uma variedade fechada (compacta sem bordo) $M$. Suponhamos que existe uma classe $C \in H^{1}(M, \mathbb{R})$ tal que para qualquer $\mu \in \mathcal{P}_{\varphi}$ temos $A_{\mu}(C)>0$. Então existe uma seção transversal $\Sigma$ ao fluxo $\varphi^{t}$.

Primeiro precisamos algumas definições e resultados. Seja $g: M \rightarrow S^{1}$ uma aplicação contínua.

Definição 2.18. Dizemos que uma função $g$ é diferenciável com respeito ao fluxo $\varphi^{t}$ ou $C^{1}$ ao longo das órbitas de $\varphi^{t}$ quando o $\lim _{t \rightarrow 0} \frac{1}{t} \Delta g(p, t)$, que denotamos por $g^{\prime}(p)$, existe para cada $p \in M$ e $g^{\prime}: M \rightarrow \mathbb{R}$ é uma aplicação contínua.

Lema 2.19. Suponhamos que g é $C^{1}$ ao longo da órbita e que $g^{\prime}$ é sempre positivo. Então o conjunto $\Sigma=\left\{x \in M: g(x)=0 \in S^{1}\right\}$ é uma seção transversal global para o fluxo.

Demonstração. Claramente $\Sigma$ é transversal ao fluxo.

Agora desde que $g^{\prime}(x)>0$, para $\epsilon>0$, temos que para qualquer ponto $p \in M$ existe $t \in \mathbb{R}$ tal que $0 \leq t \leq \frac{1}{\epsilon}$ tal que $\varphi^{t}(p) \in \Sigma$.

Teorema 2.20. Suponhamos que existem números reais $T>0$ e $\epsilon>0$ tais que

$$
\frac{1}{T} \Delta g(p, T)>\epsilon \quad \text { para cada } p \in M
$$

Então o fluxo $\varphi^{t}$ admite uma seção transversal global.

Demonstração. Considerando $S^{1}=\mathbb{R} / \mathbb{Z}$ como grupo aditivo, podemos definir uma aplicação $\bar{g}: M \rightarrow S^{1}$ pondo

$$
\bar{g}(p)=g(p)+\frac{1}{T} \int_{0}^{T} \Delta g(p, s) d s,
$$

onde a soma no segundo membro é a soma em $S^{1}$.

Provaremos que $\bar{g}$ é $C^{1}$ ao longo das órbitas de $\varphi^{t}$ e que $\bar{g}^{\prime}(p)>0, \forall p \in M$. Portanto, pelo Lema 2.19, a demonstração estará concluída. De fato, como 


$$
\begin{aligned}
\Delta \bar{g}(p, t) & =\Delta g(p, t)+\frac{1}{T}\left(\int_{0}^{T} \Delta g\left(\varphi^{t}(p), s\right) d s-\int_{0}^{T} \Delta g(p, s) d s\right) \\
& =\Delta g(p, t)+\frac{1}{T}\left(\int_{0}^{T} \Delta g(p, s+t) d s-\int_{0}^{T} \Delta g(p, t) d s-\int_{0}^{T} \Delta g(p, s) d s\right) \\
& =\frac{1}{T}\left(\int_{T}^{T+t} \Delta g(p, s) d s-\int_{0}^{t} \Delta g(p, s) d s\right),
\end{aligned}
$$

obtemos que

$$
\lim _{t \rightarrow 0} \frac{1}{t} \Delta \bar{g}(p, t)=\frac{1}{T} \Delta g(p, T)>\epsilon,
$$

$\operatorname{assim} \bar{g}$ é $C^{1}$ ao longo das órbitas de $\varphi^{t}$ e $\bar{g}^{\prime}(p)>0, \forall p \in M$ como queríamos mostrar.

A seguir veremos um exemplo onde calcularemos $A_{\mu_{p, T}}$, que será utilizado no seguinte capítulo.

Exemplo 2.21. Seja $\gamma$ uma órbita periódica de período $T$ que passa pelo ponto $p \in M$ e consideremos as sequências $p_{n}=p$ e $T_{n}=n T$. Observemos que $p_{n} \rightarrow p$ e $T_{n} \rightarrow \infty$. Dado $[g] \in\left[M, S^{1}\right]$, temos que

$$
\Delta g\left(p, T_{n}\right)=n \Delta g(p, T)
$$

pela Proposição 2.10 ii). Assim,

$$
\begin{aligned}
A_{\mu_{p_{n}, T_{n}}}([g]) & =\frac{1}{T_{n}} \Delta g\left(p_{n}, T_{n}\right) \\
& =\frac{1}{T} \Delta g(p, T) .
\end{aligned}
$$

Por outro lado, usando a Proposição 2.7 e definindo o homomorfismo $[\gamma]:\left[M, S^{1}\right] \rightarrow \mathbb{R}$ como $[\gamma]([g])=\Delta g(p, T)$, temos:

$$
A_{\mu_{p, T}}=\frac{[\gamma]}{T}
$$

Demonstração do Teorema 2.17 Primeiramente suponhamos que $C$ é um elemento inteiro de $H^{1}(M, \mathbb{R})$, ou seja $C \in H^{1}(M, \mathbb{Z})$. Então, pelo item i) da Proposição 2.8 , seja $g: M \rightarrow S^{1}$ o representante da classe $C$. Por contradição, vamos supor que o fluxo $\varphi^{t}$ não admite uma seção transversal global. Então do Teorema 2.20 segue que existe uma sequência de pontos $\left\{p_{n}\right\}$ em $M$ e $T_{n} \rightarrow \infty$ tal que

$$
\frac{1}{T_{n}} \Delta g\left(p_{n}, T_{n}\right) \rightarrow b \text { para algum } b \leq 0
$$


Agora passando a uma subsequência, se necessário, obtemos uma medida em $\mathcal{P}_{\varphi}$ dada por

$$
\mu=\lim _{n \rightarrow \infty} \frac{1}{T_{n}} \mu_{p_{n}, T_{n}} .
$$

Logo, pelo Lema 2.16, temos que $A_{\mu}([g])=b$ contradizendo nossa hipótese. Esta contradição prova o Teorema, no caso em que $C$ é uma classe inteira.

Agora consideremos o caso geral. Se a classe $C$ satisfaz as hipóteses do teorema, então, como $\left\{A_{\mu} \mid \mu \in \mathcal{P}_{\varphi}\right\}$ é compacto, todas as classes suficientemente próximas de $C$ também satisfazem esta condição. Logo podemos escolher uma classe racional $C^{\prime}$ e fazer o produto desta por um inteiro apropriado para obter uma classe inteira. Isto termina a prova pois neste caso o teorema já foi provado.

Proposição 2.22. Seja $\varphi^{t}$ um fluxo sobre uma variedade compacta $M$ e seja $\pi: \widehat{M} \rightarrow M$ um recobrimento finito. Denotemos por $\widehat{\varphi}^{t}$ o levantamento de $\varphi^{t}$ a $\widehat{M}$. Então $\varphi^{t}$ admite uma seção transversal global se e somente se $\widehat{\varphi}^{t}$ admite uma seção transversal global.

Demonstração. Claramente se $\varphi^{t}$ admite uma seção transversal global então $\widehat{\varphi}^{t}$ também admite uma seção transversal global. Suponhamos que $\widehat{\varphi}^{t}$ possui uma seção transversal global $\Sigma$ em $\widehat{M}$. Afirmamos que se $x \in \Sigma$ então existe $t_{0}>0$ tal que $\widehat{\varphi}^{t_{0}}(x) \in \Sigma$, caso contrário, o $\omega$-limite da órbita de $x$ (que é invariante pelo fluxo) seria disjunto de $\Sigma$ contradizendo a definição de seção transversal global. Consequentemente, existe uma aplicação $t: \Sigma \rightarrow \mathbb{R}^{+}$de classe $C^{1}$ tal que $\widehat{\varphi}^{t(x)}(x) \in \Sigma$ e $\widehat{\varphi}^{s}(x) \notin \Sigma$ se $0<s<t(x)$. Esta aplicação nos permite definir o difeomorfismo de primeiro retorno

$$
F: \Sigma \rightarrow \Sigma \text { pondo } F(x)=\widehat{\varphi}^{t(x)}(x)
$$

Reparametrizando o tempo, se necessário, podemos supor que $t(x)=1$ para cada $x \in \Sigma$. Isto nos permite definir $\widehat{g}: \widehat{M} \rightarrow S^{1}$ uma fibração sobre $S^{1}$ de classe $C^{1}$ ao longo das órbitas de $\widehat{\varphi}^{t}$ e tal que $\widehat{g}^{\prime}$ é sempre positiva.

Definimos a aplicação $g: M \rightarrow S^{1}$ por $g(x)=\widehat{g}\left(x_{1}\right)+\widehat{g}\left(x_{2}\right)+\cdots+\widehat{g}\left(x_{r}\right)$, onde $\left\{x_{1}, \ldots, x_{r}\right\}$ é a imagem inversa de $x$ pela aplicação de levantamento $\pi$. Daqui temos que $g$ é $C^{1}$ ao longo das órbita de $\varphi^{t}$ e $g^{\prime}$ é sempre positiva. Assim pelo Lema 2.19, o fluxo $\varphi^{t}$ admite uma seção transversal global. 


\section{Capítulo 3 \\ Grupo fundamental solúvel e Existência de seção transversal global: Um Teorema de Plante}

Neste capítulo estabeleceremos um Teorema de classificação, obtido por J. Plante em [12], para fluxos de Anosov de codimensão um sobre variedades diferenciáveis de classe $C^{\infty}$ e dimensão maior do que 3, com grupo fundamental solúvel. Mais precisamente, nosso objetivo é mostrar o seguinte resultado.

Teorema 3.1. Qualquer fluxo de Anosov de codimensão um sobre uma variedade diferenciável compacta $M$ de dimensão maior do que 3 e de classe $C^{\infty}$ com grupo fundamental solúvel é topologicamente conjugado à suspensão de um automorfismo hiperbólico do toro.

Primeiramente estabelecemos algumas definições e resultados. Um difeomorfismo de Anosov diz-se de codimensão um se a folheação estável ou a instável é de codimensão um. Definição 3.2. Sejam $f_{1}, f_{2}$ difeomorfismos sobre as variedades $N_{1}, N_{2}$ respectivamente. Dizemos que $f_{1}$ e $f_{2}$ são topologicamente conjugados ( $C^{r}$ conjugados) se existe um homomorfismo $\left(C^{r}\right.$ difeomorfismo) $h: N_{1} \rightarrow N_{2}$ tal que $f_{2} \circ h=h \circ f_{1}$.

Os difeormorfismos de Anosov de codimensão foram completamente classificados por Franks-Newhouse [6]-[11]. Mais precisamente, temos o seguinte Teorema.

Teorema 3.3 (Franks-Newhouse). Um difeomorfismo de Anosov de codimensão um sobre uma variedade fechada $N$ é topologicamente conjugado a um automorfismo hiperbólico do toro. 
Grupo fundamental solúvel e Existência de seção transversal global: Um Teorema de 26

Plante

A estratégia para mostrar o Teorema 3.1 será a seguinte. Primeiramente, mostraremos que a condição do critério de Schwartzman é satisfeita, isto implicará que o fluxo admite uma seção transversal global $\Sigma$. Isto significa que a aplicação de primeiro retorno $T$ : $\Sigma \rightarrow \Sigma$ é um difeomorfismo e o fluxo é topologicamente equivalente à suspensão de $T$. O fato do fluxo $\varphi^{t}$ ser de Anosov implica que a aplicação de primeiro retorno $T$ : $\Sigma \rightarrow \Sigma$ é um difeomorfismo de Anosov, logo, pelo Teorema de Franks-Newhouse, $T$ é topologicamente conjugado a um automorfismo hiperbólico do toro. Consequentemente, $\varphi^{t}$ é topologicamente equivalente à suspensão de um automorfismo hiperbólico do toro.

\subsection{Demonstração do Teorema Principal}

Como mencionado acima, para mostrar o Teorema 3.1, é suficiente mostrar o seguinte.

Lema 3.4. O fluxo $\varphi^{t}$ admite seção transversal global.

Observação 3.5. Sendo $\varphi^{t}$ um fluxo de Anosov de codimensão um sobre uma variedade de dimensão maior do que 3, temos as seguintes observações:

(1) Podemos supor que a folheação centro estável é transversalmente orientável. De fato pelo Teorema 2.22, o fluxo $\varphi^{t}$ admite uma seção transversal se e somente se um levantamento $\widehat{\varphi}^{t}$ finito do fluxo admite uma seção transversal global. Assim é suficiente passar ao recobrimento duplo orientável, caso a folheação não seja transversalmente orientável.

(2) As folhas das folheações centro estável e centro instável são densas em $M$. Isto é consequência do Teorema 1.27 .

(3) A holonomia das folhas da folheação centro estável é trivial ou tem um ponto fixo isolado. Isto é consequência direta do fato de o fluxo ser Anosov.

Seja $\mathrm{Aff}_{+}(\mathbb{R})$ o grupo das transformações afins da reta que preservam orientação. Isto é, cada elemento $h$ em $\mathrm{Aff}_{+}(\mathbb{R})$ é da forma $h(x)=a x+b$ com $a>1$. Tendo em consideração os itens anteriores e o fato do grupo fundamental da variedade ser solúvel, um resultado de J. Plante (Theorem 6.2 de [12]) garante que:

(4) A folheação centro estável $\mathcal{W}^{s}$ é topologicamente conjugada a uma folheação transversalmente afim. Isto é obtemos um par de aplicações equivariantes

$$
(h, D):\left(\pi_{1}(M), \tilde{M}\right) \rightarrow\left(\mathrm{Aff}_{+}(\mathbb{R}), \mathbb{R}\right)
$$

onde D é uma submersão topológica. 
Para cada $\gamma \in \pi_{1}(M)$ a aplicação $h(\gamma)$ é uma transformação afim da forma:

$$
x \mapsto a(\gamma) x+b(\gamma) \operatorname{com} a(\gamma)>0
$$

Logo podemos definir o homomorfismo

$$
k: \pi_{1}(M) \rightarrow \mathbb{R} \text { como } k(\gamma)=\ln (a(\gamma))
$$

Usando os seguintes isomorfismos conhecidos, os quais podem ser encontrados em [21],

$$
\operatorname{Hom}\left(\pi_{1}(M), \mathbb{Z}\right) \cong\left[M, S^{1}\right] \cong H^{1}(M, \mathbb{Z})
$$

além disso sabemos que:

$$
\operatorname{Hom}\left(\pi_{1}(M), \mathbb{R}\right) \cong H^{1}(M, \mathbb{R})
$$

e assim temos que o homomorfismo $k$ define uma classe em $H^{1}(M, \mathbb{R})$ a qual denotaremos por $C$.

Demonstração do Lema 3.4 Usando o critério de Schwartzman é suficiente mostrar que $A_{\mu}(C)>0$ para toda $\mu$ medida de probabilidade invariante pelo fluxo.

A partir de agora nos dedicaremos a mostrar que $A_{\mu}(C)>0$ para toda $\mu$ medida de probabilidade invariante pelo fluxo. Seja $\gamma$ uma órbita periódica do fluxo $\varphi^{t}$ passando pelo ponto $p$ com período $T$ denotemos por $\mu(\gamma)$ a medida $\mu_{p, T}$ definida na Seção 2.2.

Lema 3.6. Seja $\gamma$ uma órbita periódica do fluxo $\varphi^{t}$, com a correspondente medida de probabilidade $\mu(\gamma)$ invariante pelo fluxo. Então temos que $A_{\mu(\gamma)}(C)>0$.

Demonstração. A classe de homologia $A_{\mu(\gamma)}$ é dada por $[\gamma] / T$, ver Exemplo 2.21. Mas, como $H_{1}(M, \mathbb{R}) \cong \operatorname{Hom}\left(H^{1}(M, \mathbb{R}), \mathbb{R}\right)$, então $A_{\mu(\gamma)}(C)$ não é mais do que a avaliação de $C$ em $[\gamma] / T$, isto é $A_{\mu(\gamma)}(C)=\frac{1}{T} k\left(\gamma_{0}\right)$, onde $\gamma_{0}$ é um elemento de $\pi_{1}(M)$ homotópico a $\gamma$. Logo $T A_{\mu(\gamma)}(C)$ é exatamente o logaritmo da parte linear da holonomia da folha da folheação $\mathcal{W}^{s}$ ao longo da órbita $\gamma$. Agora a folheação instável $\mathcal{W}^{u u}$ é unidimensional e transversal $\mathcal{W}^{s}$. Logo pela propriedade expansiva do fluxo $\varphi^{t}$ ao longo das folhas de $\mathcal{W}^{\text {uu }}$ temos que $A_{\mu(\gamma)}(C)>0$.

A propriedade expansiva do fluxo $\varphi^{t}$ ao longo das folhas de $\mathcal{W}^{u u}$ sugere que $A_{\mu}(C)>0$ para toda medida de probabilidade $\mu$ invariante pelo fluxo. No entanto mostrar isto de maneira direita é difícil. Para mostrar isto consideraremos uma aproximação de $C$ pelas classes racionais em $H^{1}(M, \mathbb{Q})$ e usaremos partições de Markov associadas a um fluxo de Anosov. 
Grupo fundamental solúvel e Existência de seção transversal global: Um Teorema de

Seja $\mathcal{R}=\left\{R_{i}\right\}_{i=1}^{r}$ uma partição de Markov associada ao fluxo $\varphi^{t}$. Como em 1.2.1 denotamos por $|\mathcal{R}|=\cup_{i=1}^{r} R_{i}$, e por $f:|\mathcal{R}| \rightarrow|\mathcal{R}|$ a aplicação de primeiro retorno, com tempo de primeiro retorno $\tau:|\mathcal{R}| \rightarrow \mathbb{R}$. Existem números reais positivos $\tau_{0}$ e $\tau_{1}$ tais que $\tau_{0} \leq \tau(x) \leq \tau_{1}$ para qualquer $x \in|\mathcal{R}|$.

Observação 3.7. Seja $\Gamma=\left\{\gamma_{k} \mid 1 \leq k \leq m\right\}$ a familia de todas as órbitas periódicas de $\varphi^{t}$ associadas com as sequencias cíclicas minimais. Seja $s_{k}$ o período de $\gamma_{k}$. Pelo Lema 3.8 , temos que $A_{\mu\left(\gamma_{k}\right)}(C)>0$ para todo $1 \leq k \leq m$. Seja $C^{\prime} \in H^{1}(M, \mathbb{Q})$ uma classe suficientemente proxima de $C$, então $A_{\mu\left(\gamma_{k}\right)}\left(C^{\prime}\right)>0$ para todo $1 \leq k \leq m$. Podemos supor que $C^{\prime}$ é uma classe inteira, se necessário multiplicamos esta por inteiros apropriados.

Seja $g: M \rightarrow S^{1}$ a aplicação a qual representa esta classe integral. Então temos que existe $\eta>0$ tal que

$$
A_{\mu\left(\gamma_{k}\right)}([g])>\eta, \text { para } k \text { tal que } 1 \leq k \leq m
$$

Nosso objetivo é mostrar que $g$ satisfaz a condição do Teorema 2.20. Assim mostraremos o seguinte resultado.

Lema 3.8. Para qualquer $T>0$ suficientemente grande, existe $\epsilon>0$ tal que

$$
\frac{1}{T} \Delta g(x, T)>\epsilon, \forall x \in M
$$

Demonstração. Imediatamente devemos mostrar o lema para $x \in|\mathcal{R}|$. Seja $T_{0}$ un número positivo a ser definido posteriormente. Para um ponto $x \in|\mathcal{R}|$, seja $\left\{i_{n}\right\}$ uma sequencia admissível associado a este. Isto é, existe uma sequencia $\left\{t_{n}\right\}$ com

$$
t_{0}=0, \tau_{0} \leq t_{n+1}-t_{n} \leq \tau_{1}, x_{n}=\varphi^{t_{n}}(x) \in R_{i_{n}} .
$$

Estamos interessados nas sequências $\left\{x_{n}\right\}_{n=0}^{N}$ e $\left\{t_{n}\right\}_{n=0}^{N}$ com $t_{N} \geq T_{0}$. Verificaremos o lema para $T=t_{N}$.

Agora trocando a função $g: M \rightarrow S^{1}$, se for necessário, na sua classe de homotopia, podemos assumir que $g$ é constante sobre cada retângulo $R_{i_{n}}$. Para cada $n$ tal que $0 \leq n \leq N$, o tempo de primeiro retorno $\tau$ e a aplicação de primeiro retorno $f$ é contínua sobre $R_{i_{n}} \cap f^{-1}\left(R_{i_{n+1}}\right)$. Definimos o número $\Delta_{n} g=\Delta g(x, \tau(x))$ que é constante para cada $x \in R_{i_{n}} \cap f^{-1}\left(R_{i_{n+1}}\right)$. Temos $\left|\Delta_{n} g\right| \leq L$ para alguma constante $L$ que depende só de $\mathcal{R}$ e g. Temos

$$
\Delta g\left(x, t_{N}\right)=\sum_{n=0}^{N-1} \Delta_{n} g .
$$


Da sequência $\mathbf{i}=\left\{i_{0}, i_{1}, \cdots, i_{N}\right\}$, podemos escolher um número $n_{1}$ e $q_{1}>0$ tal que $i_{n_{1}}=i_{n_{1}+q_{1}}$ e que $i_{n_{1}}, i_{n_{1}+1}, \cdots, i_{n_{1}+q_{1}-1}$ são todos distintos. Seja $\mathbf{j}_{1}=\left\{i_{n}, \cdots, i_{n_{1}+q_{1}-1}\right\}$ a correspondente sequência cíclica minimal admissível. Então $\mathbf{j}_{1}$ corresponde a uma órbita periódica $\gamma_{k_{1}}\left(\right.$ de período $\left.s_{k_{1}}\right)$ na família $\Gamma$. Assim temos

$$
\begin{gathered}
\sum_{\nu=n_{1}}^{n_{1}+q_{1}-1} \Delta_{\nu} g=s_{k_{1}} A_{\mu\left(\gamma_{k_{1}}\right)}([g]) \\
\frac{\tau_{0}}{\tau_{1}} \leq \frac{t_{n_{1}+q_{1}}-t_{n_{1}}}{s_{k_{1}}} \leq \frac{\tau_{1}}{\tau_{0}}
\end{gathered}
$$

Agora seja

$$
\mathbf{i}^{\prime}=\left\{i_{0}, \cdots, i_{n_{1}-1}, i_{n_{1}+q_{1}}, \cdots, i_{N}\right\} .
$$

i' é também admissível. Outra vez escolhemos números $n_{2}$ e $q_{2}$ e construímos uma sequência admissível $\mathbf{j}_{2}$ de $\mathbf{i}^{\prime}$, a qual corresponde a uma órbita periódica $\gamma_{k_{2}}$ de período $s_{k_{2}}$. Procedemos desta maneira, até terminar num número finito de passos. Então conseguimos órbitas periódicas $\gamma_{k_{1}}, \cdots, \gamma_{k_{l}}$, que é uma sequência admissível de tamanho menor que $r$ ( $r$ é o número de retângulos na partição de Markov $\mathcal{R}$ ).

Assim por 3.2 e 3.3 , temos

$$
\Delta g\left(x, t_{N}\right)=\sum_{j=1}^{l} s_{k_{j}} A_{\mu\left(\gamma_{k_{j}}\right)}([g])+E_{1}
$$

onde $\left|E_{1}\right| \leq r L$.

Temos também por 3.4

$$
\frac{\tau_{0}}{\tau_{1}} \leq \frac{t_{N}-E_{2}}{\sum_{j=1}^{l} s_{k_{j}}} \leq \frac{\tau_{1}}{\tau_{0}}
$$

onde $0 \leq E_{2} \leq r \tau_{1}$.

Notemos que $A_{\mu\left(\gamma_{k}\right)}=[\gamma] / s_{k}$. Assim por 3.1, temos

$$
\Delta g\left(x, t_{N}\right) \geq \eta \sum_{j=1}^{l} s_{k_{j}}+E_{1} .
$$

Por 3.5 e 3.6, obtemos

$$
\frac{1}{t_{N}} \Delta g\left(x, t_{N}\right) \geq \frac{\eta \sum_{j=1}^{l} s_{k_{j}}-\left|E_{1}\right|}{\frac{\tau_{1}}{\tau_{0}} \sum_{j=1}^{l} s_{k_{j}}+E_{2}}
$$


Grupo fundamental solúvel e Existência de seção transversal global: Um Teorema de 30

Donde deduzimos que

$$
\frac{1}{t_{N}} \Delta g\left(x, t_{N}\right) \geq \frac{\tau_{0} \eta}{\tau_{1}}-\frac{C}{t_{N}}
$$

onde $C=C\left(L, r, \tau_{0}, \tau_{1}\right)$ não depende da escolha de $x$ ou $t_{N}$. Portanto se podemos escolher $T_{0}$ tal que

$$
T_{0} \geq \frac{2 \tau_{1} C}{\tau_{0} \eta}
$$

então temos

$$
\frac{1}{t_{N}} \Delta g\left(x, t_{N}\right) \geq \frac{\tau_{0} \eta}{2 \tau_{1}}
$$

Se tomamos $t_{N} \geq T_{0}$ temos a prova completa 


\section{Referências Bibliográficas}

[1] Anosov, D. V., Geodesic flows on closed Riemann manifolds with negative curvature. Proceedings of the Steklov Institute of Mathematics, American Mathematical Society, Providence R.I., 1969.

[2] Bonatti, Christian and Langevin, Rémi, Un exemple de flot d'Anosov transitif transverse à un tore et non conjugué à une suspension, Ergodic Theory and Dynamical Systems, 14, 633-643, 1994.

[3] Bowen, Rufus, Symbolic dynamics for hyperbolic flows, Proceedings of the International Congress of Mathematicians , 2, 299-302, Canad. Math. Congress, Montreal, Que., 1975.

[4] Camacho, César and Lins Neto, Alcides, Teoria geométrica das folheações. Projeto Euclides, Instituto de Matemática Pura e Aplicada, Rio de Janeiro, 1979.

[5] Candel, Alberto and Conlon, Lawrence, Foliations I. Graduate Studies in Mathematics, American Mathematical Society, Providence R.I., 2000.

[6] Franks, John, Anosov diffeomorphisms on tori, Transactions of the American Mathematical Society, 145, 117-124, 1969.

[7] Franks, John and Williams, Bob, Anomalous Anosov flows, Global theory of dynamical systems, 819, 158-174, Springer, Berlin, 1980.

[8] Greenberg, Marvin J. and Harper, John R., Algebraic topology. Mathematics Lecture Note Series, Benjamin/Cummings Publishing Co. Inc. Advanced Book Program, Reading, Mass., 1981.

[9] Hirsch, Morris W. and Pugh, Charles C., Stable manifolds and hyperbolic sets, Global Analysis (Proc. Sympos. Pure Math., XIV, 133-163, Amer. Math. Soc., Providence R.I., 1970. 
[10] Katok, Anatole and Hasselblatt, Boris, Introduction to the modern theory of dynamical systems. Encyclopedia of Mathematics and its Applications, Cambridge University Press, Cambridge, 1995.

[11] Newhouse, S. E., On codimension one Anosov diffeomorphisms, American Journal of Mathematics, 92, 761-770, 1970.

[12] Plante, J. F., Solvable groups acting on the line, Transactions of the American Mathematical Society, 278, 401-414, 1983.

[13] Robinson, Clark, Dynamical systems. Studies in Advanced Mathematics, CRC Press, Boca Raton FL, 1999.

[14] Scárdua, Bruno and Morales, Carlos, Geometry, dynamics and topology of foliated manifolds. Publicações Matemáticas do IMPA, Instituto de Matemática Pura e Aplicada (IMPA), Rio de Janeiro, 2003.

[15] Schwartzman, Sol, Asymptotic cycles, Annals of Mathematics. Second Series, 66, 270-284, 1957.

[16] Smale, S., Stable manifolds for differential equations and diffeomorphisms, Topologia Differenziale, Lezione 4, 97-116, Edizioni Cremonese, Rome, 1963.

[17] Smale, S., Differentiable dynamical systems, Bulletin of the American Mathematical Society, 73, 747-817, 1967.

[18] Tamura, Itiro, Topology of foliations: an introduction. Translations of Mathematical Monographs, American Mathematical Society, Providence RI, 1992.

[19] Verjovsky, Alberto, Codimension one Anosov flows, Bol. Soc. Mat. Mexicana (2), 19, 49-77, 1974.

[20] Verjovsky, Alberto, Sistemas de Anosov. Monografías del Instituto de Matemática y Ciencias Afines, Instituto de Matemática y Ciencias Afines IMCA, Lima, 1999.

[21] Vick, James W., Homology theory. Graduate Texts in Mathematics, Springer-Verlag, New York, 1994. 